\title{
AN OVERVIEW OF GLOBULAR CLUSTER SYSTEMS IN DISTANT GALAXIES
}

\author{
William E. Harris \\ McMaster University
}

ABSTRACT: Information is currently available for globular cluster systems in almost 50 galaxies from the Milky Way to the Coma Cluster. The observed features of these systems are reviewed, with emphasis on (a) their total populations (specific frequencies), (b) spatial structures compared with the underlying halo light, (c) photometric characteristics (luminosity function and metallicity). The combined evidence suggests strongly that globular clusters are likely to have formed in a rather sharply defined epoch clearly before the bulk of the halo field stars, and that this epoch was more active in $E$ galaxies, especially those in rich environments. Finally, the special role of the supergiant E's at the centers of rich galaxy clusters is reviewed.

\section{INTRODUCTION}

In 1918, a paper by Harlow Shapley appeared in the Astrophysical Journal containing a discussion of the overall space distribution of the globular clusters in the Milky Way. For Shapley, this summary culminated years of patient accumulation of data on the Galactic globular clusters, one object after another, until a global picture of the entire halo began to emerge. But this paper can also now be seen as the first stride into a completely new realm: Shapley was the first to study a globular cluster system as a complete entity. Shapley's essential vision that the cluster system formed the skeletal outline of the entire Galaxy remains remarkably valid seventy years later.

Discovery and study of globular cluster systems (GCS's) in distant, major galaxies - M 31 and beyond - began with Hubble's work (1932). However, the basic nature of the problem from an observer's point of view is that globular clusters are very faint relative to their parent galaxies, and the galaxies concerned are very distant; so substantial progress in the observations was slow until the current decade, with the notable exception of the Virgo System survey by Hanes $(1977 a, b)$. During previous conferences in the past decade, the only reviews relevant to globular cluster systems in galaxies beyond the Local Group are those given by Hanes (1980) and by Racine (1980). It is an encouraging sign of the rapid growth of this field that a large 
TABLE I

GLOBULAR CLUSTER SYSTEMS IN GALAXIES

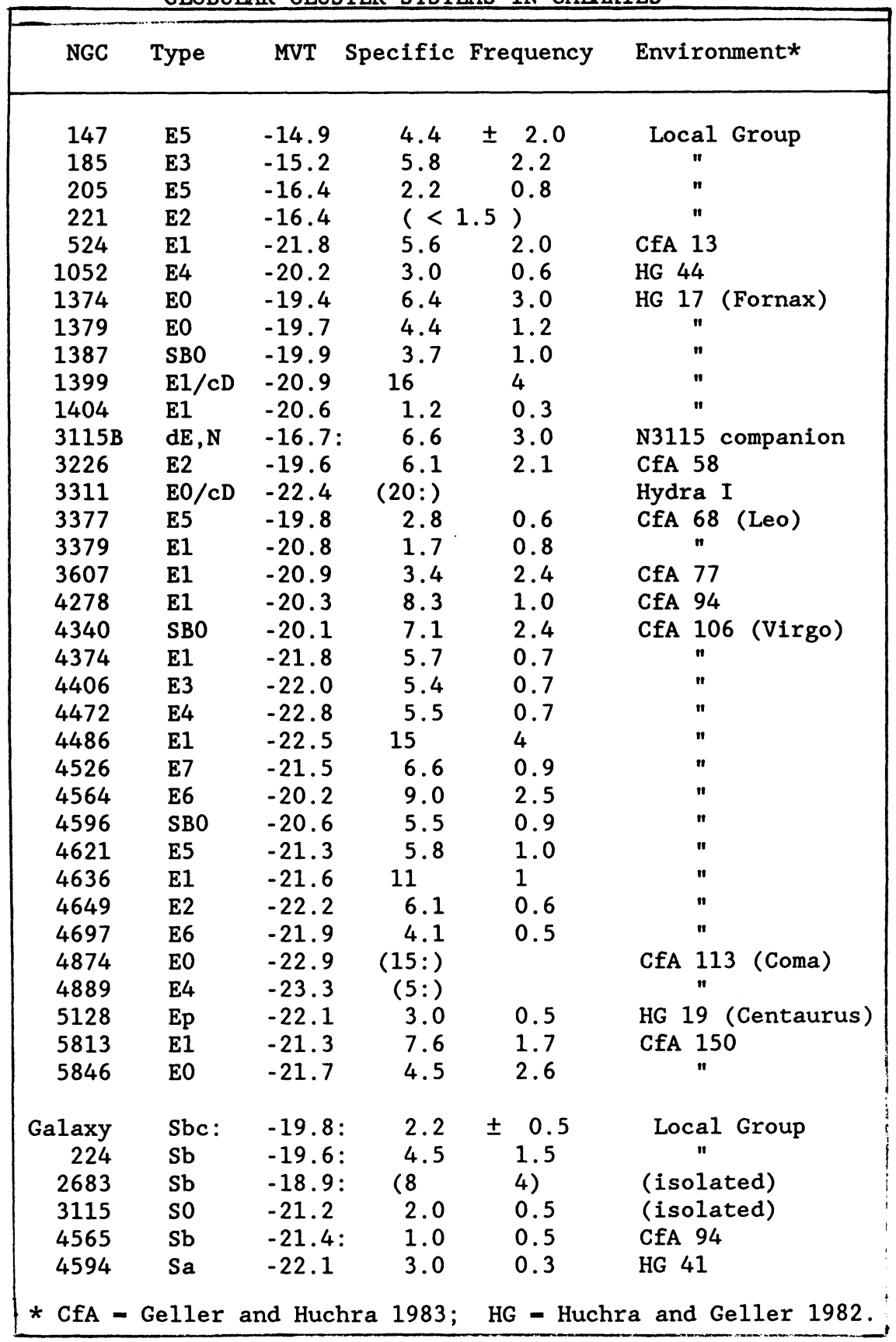


part of this Symposium contains discussions of the GCS's in numerous galaxies from the Milky Way all the way to the Coma Cluster. Most badly needed of all, strong contributions are beginning to come from the theorists on the topics of cluster formation and dynamical evolution of galactic halos. Numerous fundamental questions (How old are the clusters relative to the rest of the halo? Were the clusters responsible for "seeding" the enrichment of the entire halo? How do these factors depend on galaxy environment? What were the conditions that produced the extraordinary GCS's surrounding the supergiant ellipticals at the centers of rich clusters?) will be answerable in detail only with well constructed models that do not yet exist.

\section{TOTAL POPULATIONS AND SPECIFIC FREQUENCIES}

This review will highlight what we have learned about the overall properties of GCS's observationally since 1980 (see Harris and Racine 1979 for a literature review up to that point). In 1980, a survey program designed to investigate the GCS's in a widely chosen sample of large galaxies was begun, primarily with the wide-field prime focus camera of the CFHT (Harris and van den Bergh 1981; and later papers in that series). By counting the projected number density of starlike images around each galaxy as a function of radius (ideally until the counts drop to a constant "background" level), we derive both the total population of clusters belonging to the galaxy and their radial distribution in the halo (see, e.g., Harris 1986 or Harris and van den Bergh 1981 for a more complete discussion of the methodology). A summary list of currently available data for more than 40 GCS's is given in the adjacent table (excluding only those in Sc/Irr galaxies), and additional new ones are reported in other papers at this meeting.

The first totally new result of the survey program was a comparison of the total populations in the cluster systems, and a test of the earlier belief (Hanes 1977, Harris and Racine 1979) that the number of clusters $\mathrm{N}$ found in a galaxy was directly proportional to the total spheroidal luminosity $L$. The single clear exception known at that time was the enormously populous GCS in M 87, which was thought to be related somehow to its central location in Virgo (Harris and Smith 1976; van den Bergh 1977). A new quantity called the specific frequency, $S$, (equal to the number of clusters per unit galaxy luminosity; cf. Harris and van den Bergh 1981) was defined to help the process of comparison. The average $S$-value is plainly not uniform from one galaxy to another; that is, the strict $N(L)$ proportionality rule is followed only in a rough sense. The most obvious variations appear to be correlated with galaxy environment (Fig. 1), such that systems in rich surroundings like the Virgo and Fornax groups have a mean $S$ almost twice as large as those in the smaller, sparser groups which can be found in and around the Local Supercluster. At least part of this difference might be due to the varying importance of galaxy mergers in different environments (Harris 1981), but this approach is not likely to explain the low $\langle S\rangle$ observed for the disk galaxies in these groups too. Close but less catastrophic encounters between galaxies are 
capable of rearranging the numbers of halo stars and clusters held by big vs. small galaxies (see Muzzio et al. 1984; Muzzio 1986), but here again it is doubtful whether the full range of the observed $S$-values can be produced this way, especially the more extreme $M$ 87-type anomaly. The most likely explanation (see the more extensive discussion of alternatives by Harris 1986) may instead require that $E$ galaxies in different locations went through early cluster formation epochs of different intensities, or of different durations; and that the halos of disk galaxies were not as prolific at halo cluster formation as were ellipticals. Other clues to the GCS formation period will be added in the sections following.

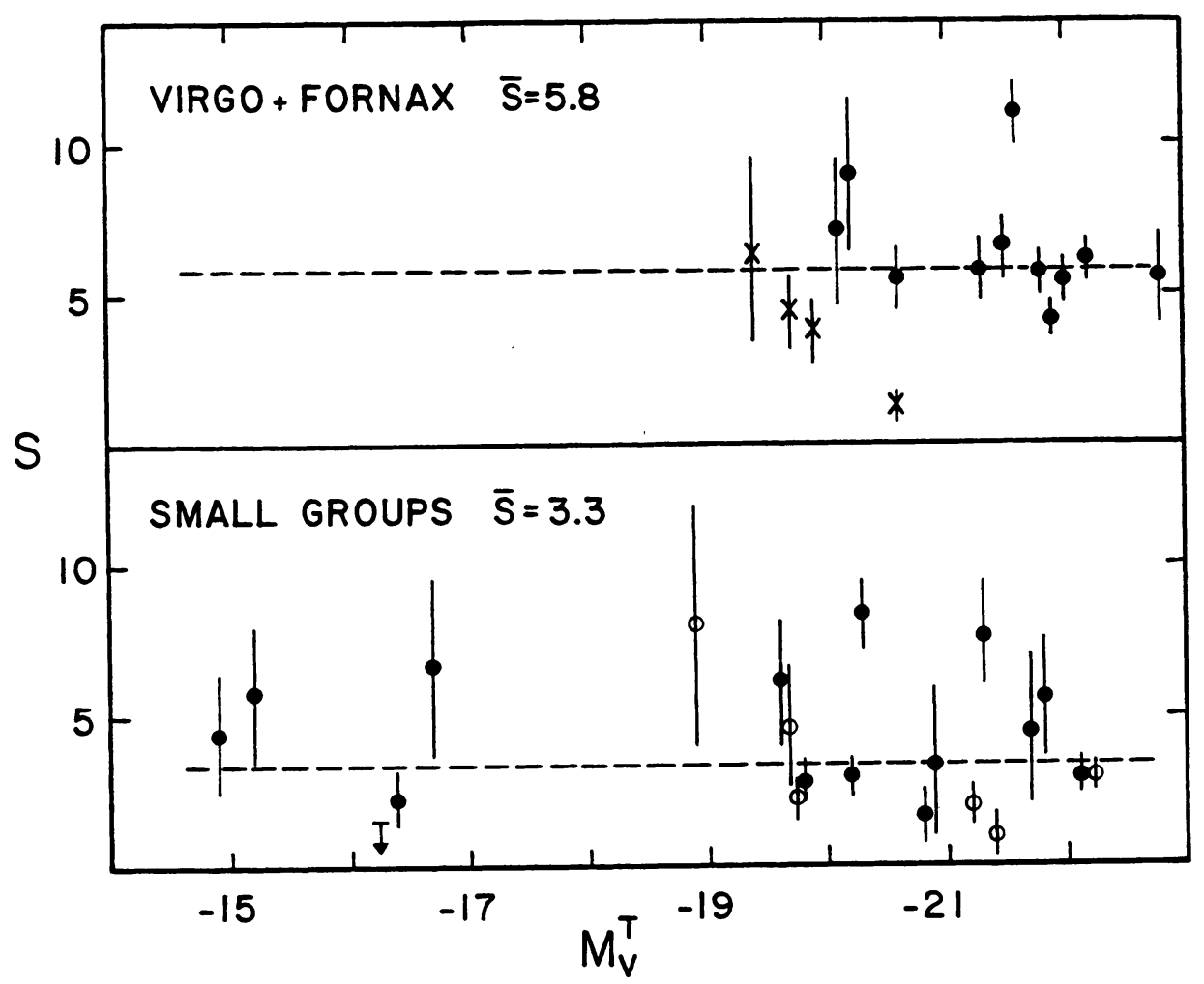

Fig. 1. Specific frequency $S$ plotted vs. galaxy luminosity $\mathbb{M}_{\checkmark}{ }^{\top}$ for 36 galaxies. Top pane1: dots represent Virgo E/SO systems, crosses show the Fornax ellipticals. Bottom panel: dots represent E/SO systems found in small galaxy groups, while open circles represent disk galaxies (in this case $M_{v}{ }^{T}$ refers to the total spheroid luminosity excluding the disk). Dashed lines show the mean (weighted inversely as the internal errors) for each group; the systems in the richer environments (at top) have a higher $\langle S\rangle$ by almost a factor of two. 


\section{SPACE DISTRIBUTIONS AND RADIAL STRUCTURES}

The classical assumption (e.g. Blaauw 1965) is that globular clusters and halo field stars are equally useful tracers of the same oldest Population II. A different view was first expressed by Harris and Racine (1979) that "... the globular cluster systems have significantly larger characteristic sizes than do the parent galaxies ... this may indicate that the clusters were formed at an earlier epoch than were the stars of the spheroidal component, i.e. at a time when the protogalactic material was less centrally condensed."

The most dramatic evidence for this distinction is probably to be found in $M 87$ itself, where we now have a projected radial profile all the way from the center out to its remotest outskirts (Fig. 2). The difference in slope between the cluster distribution and the halo surface intensity persists everywhere through the galaxy, and provides compelling evidence that, at least in $M 87$, we are looking at two

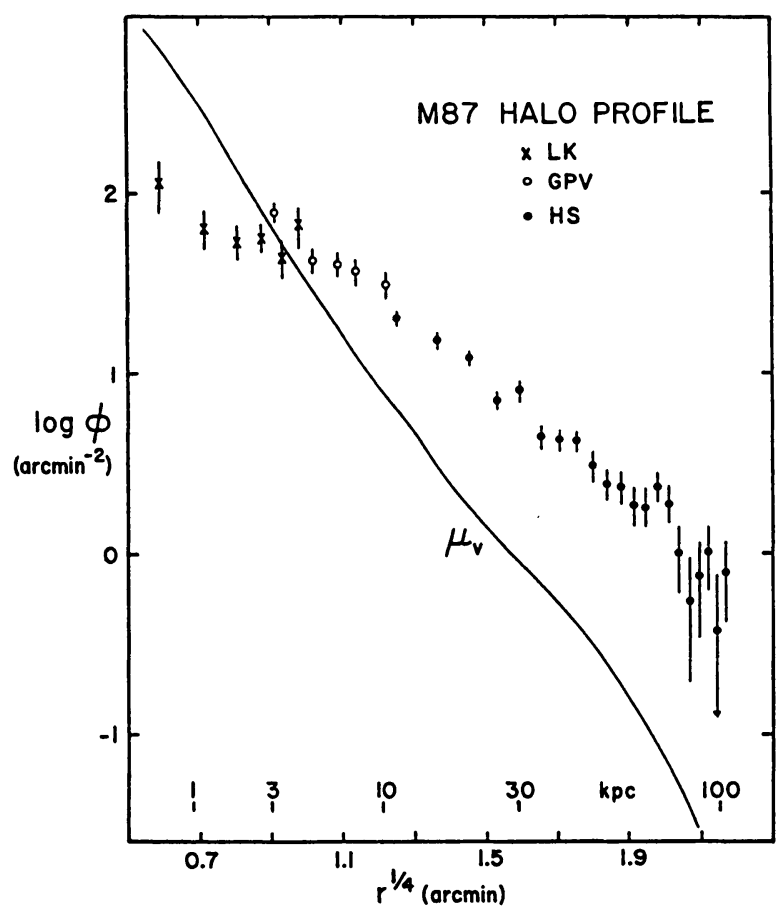

Fig. 2. Radial distribution of globular clusters in $M 87$. Here $\phi$ is the number of clusters per square arcmin brighter than $B=23.6$, versus galactocentric radius $r$ (the scale in $\mathrm{kpc}$ assumes $\mathrm{d}-16 \mathrm{Mpc}$ ). Crosses are from Lauer and Kormendy (1986); open circles from Grillmair et al. (1986); and filled circles from Harris (1986). The solid line is the V surface intensity of the M 87 halo, from Oemler (1976). 
separate populations. Several recent discussions (Lauer and Kormendy 1986; Grillmair et a1. 1986; Harris 1986) make it extremely doubtful that such an extended GCS profile could be generated artificially by an ongoing process of dynamical evolution. Preferential destruction of the inner clusters by dynamical friction operates effectively only within 1-2 kpc of the center; and adding large numbers of clusters in the outer parts due to galaxy-galaxy interactions would add similar proportions of halo field stars as well, so that both types of populations would maintain the same radial profiles to first order.

We should, of course, keep in mind that the M 87 GCS is an anomalous case. We need to search for a general process (as opposed to something designed especially for one object) only if we find the same pattern showing up elsewhere. However, the same radial distribution is found in $M$ 49, a Virgo galaxy of the same size and structure as $M 87$ but well away from the Virgo center and with a thoroughly "normal" specific frequency (Harris 1986). It should also be emphasized that, because the $M 87$ and $M 49$ radial distributions are nearly identical in shape (Harris 1986), the basic M 87 "anomaly" of a specific frequency 3 times higher than average exists everywhere through its halo. Thus we cannot solve this anomaly by, for example, adding in large numbers of clusters only in the outer parts of the M 87 halo (either by formation or by tidal accretion) while leaving the inner halo alone. Something to do with the formation of the parent galaxy must be involved in making the GCS more spatially extended than the main halo.

Interestingly, there are two known cases of GCS's in central cD-type galaxies: NGC 1399 in Fornax (Hanes and Harris 1986b) and NGC 3311 in Hydra I (Harris et al. 1983). In both of these, there is good agreement between the halo light profile and the GCS profile - not because the GCS is any different from the M 87 and M 49 cases, but because the $c D$ envelope is more extended than a normal elliptical. For the more normal E's, a few less certain cases are known (cf. Harris and van den Bergh 1981, and Hanes 1977a) in which the GCS appears to have a broader space distribution than the galaxy light; significantly, no $E$ galaxy is known where the reverse is true.

The data for GCS's in two large disk galaxies, NGC 4594 (Harris et al. 1984) and NGC 3115 (Hanes and Harris 1986a) are informative in a different way. Here, the isophotes for each galaxy are quite plainly flattened along the major axis over the spatial region where globular clusters are observed in large numbers $(r<30 \mathrm{kpc})$. Yet in both cases, the clusters lie in a distribution that is (within the count statistics) indistinguishable everywhere from spherical; that is, the cluster system is rounder than the underlying halo. Acceptable agreement between the radial profiles of the GCS and the halo light is obtained only if the surface intensity along the minor axis is used. Interestingly, H. Harris et al. (1987) report that in another edge-on Sa disk system, NGC 7814, the GCS is flattened much like the spheroid light (by rotation?), yet is more spatially extended than the spheroid! 
One last, less certain result has emerged recently in which halo cluster systems seem to mimic a trend with luminosity that the galaxies themselves follow. This is that the GCS appears to become less centrally concentrated with increasing galaxy luminosity (Harris 1987). In terms of a power-law index $a=\Delta \log \sigma / \Delta \log r$ where $\sigma$ is the projected number of clusters per unit area around the galaxy, the rate of decline of $a$ is about 0.3 (toward lower absolute value) for every 1 -magnitude increase in galaxy luminosity. Both the slope of this trend, and the scatter around it, strongly resemble plots of profile slope vs. luminosity for the galaxies themselves (e.g. Strom and Strom 1978), but just displaced toward lower central concentration by $\Delta a-$ 0.4 .

\section{LUMINOSITY FUNCTIONS AND PHOTOMETRIC COLORS}

Globular clusters have long been potentially attractive as extragalactic distance indicators, since: (a) they are as luminous as other conventionally used standard candles such as Cepheids, HII regions, supergiants, or novae; (b) they do not suffer from the internal reddening and crowding that affect all Population I indicators; (c) they do not require the long time series of repeated measurements that all variable stars do; and (d) they afford the chance to construct a pure Population II distance scale related ultimately to the assumed luminosity of the RR Lyrae stars. Nevertheless, they will become truly useful as standards only if we can show that they follow a 'universal' luminosity distribution in different galaxies (or, if their intrinsic LF does differ from one type of galaxy to another, we at least need to understand exactly how these differences behave systematically).

The assumption is often made (e.g. Hanes 1977a; Harris and Racine 1979; van den Bergh 1985) that the LF for globular clusters is Gaussian in $n\left(M_{v}\right)$. This use of a normal distribution is (so far) strictly an empirical approximation with no rigorous theoretical basis, but there are more reasons for using it than just numerical convenience. Fig. 3 shows the observed LF for M 87 (van den Bergh et al. 1985) combined with the sum of 6 large Virgo ellipticals (Hanes 1977a) to produce a combined LF spanning almost 5 magnitudes and apparently reaching just past the peak or "turnover" point. It is clear that a straight power law - as has sometimes been proposed (Tremaine 1976; Racine 1980) will not match this distribution. The normal curve superimposed on the M 87/Virgo data has a dispersion of $1.4 \pm 0.1 \mathrm{mag}$, and the peak near $B$ $=24.7$ corresponds to a Virgo distance of $16 \mathrm{Mpc}$ (or $\mathrm{H}_{\mathrm{o}}=80 \mathrm{~km} / \mathrm{s} / \mathrm{Mpc}$ ). This fit, or others close to it (e.g. Hanes 1987) clearly provides a satisfactory description of the Virgo data.

It is not yet clear just how similar to the $M 87 /$ Virgo distribution the LF's of globular cluster systems in other galaxies 
actually are. The reasons for this are that a small sample size obscures the intrinsic properties of the LF (in the case of our Local Group members, including the Milky Way), or that insufficiently deep or complete photometry is available to reach close to the turnover point (in the case of more distant major ellipticals or spirals). However, at least the intrinsic dispersion of the LF is known to be similar enough in other galaxies to keep them competitive as an interesting distance indicator: for the Milky Way and M 31, van den Bergh (1985) finds $\sigma=1.2$ to 1.3 magnitudes, and for NGC 5128, G. Harris et al. (1984) estimate $\sigma=1.4 \mathrm{mag}$. Deep CCD photometry now in progress for the GCS's around 3 more Virgo giant ellipticals and several other small ones in other groups (Harris and Allwright 1987; Hanes and Harris 1987) should soon help answer the question whether the dispersion or peak luminosity of the LF vary significantly between $E$ galaxies. If both $\sigma$

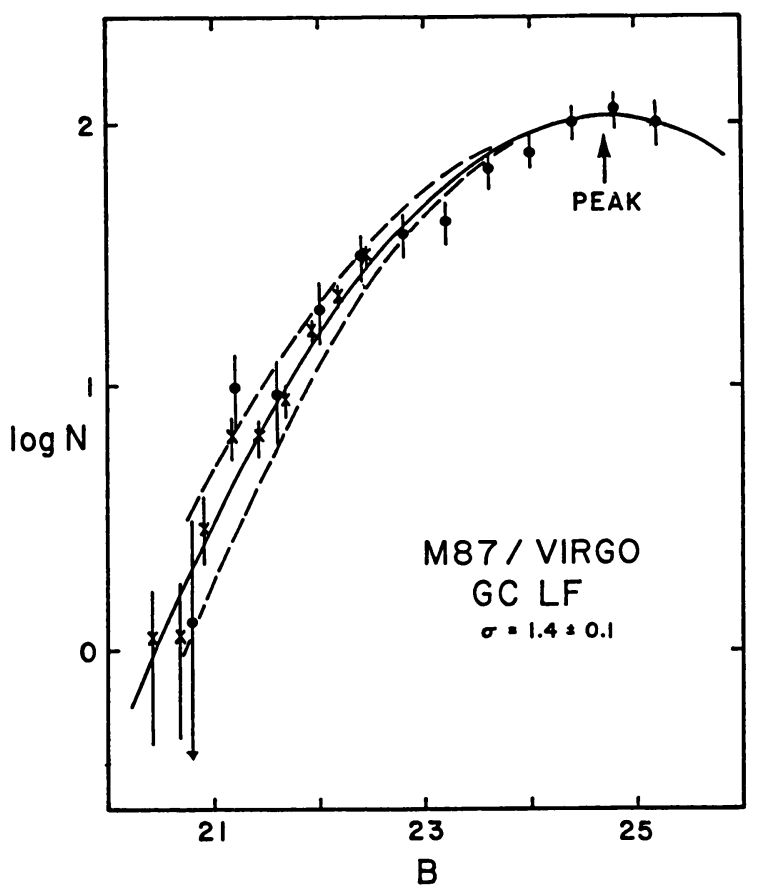

Fig. 3. Luminosity distribution of the globular clusters in the Virgo ellipticals. Crosses represent the sum of globular cluster populations in 6 large Virgo E's, from Hanes (1977a); dots are the faint $M 87$ sample measured by van den Bergh et al. (1985). A Gaussian curve with peak ("turnover") at $B=24.7$ and dispersion $1.4 \mathrm{mag}$ (solid line) is shown fitted to the data, along with similar curves for dispersions of 1.3 and $1.5 \mathrm{mag}$ (dashed lines). 
and $M_{B}$ (peak) turn out to be sensibly constant over the range of parent galaxies that have already been observed (more than a factor of 1000 in total luminosity, and over Hubble types from $\mathrm{E}$ to $\mathrm{Sb}$ ), then the GCS's will also provide important constraints on modelling the protogalactic epoch in which they were formed. It may be that the clusters belonged to such an early stage of the protogalactic collapse that their mass spectrum of formation was relatively uninfluenced by the type or size of galaxy that would eventually emerge (but see Fall and Rees 1985).

In terms of photometric colors and metallicity characteristics, it has been known for some time (Hanes 1977b, Harris and Racine 1979) that clusters in many galaxies are roughly similar, with a slight trend for those in bigger galaxies to be somewhat more metal-rich (redder). An important new step was taken with the work of Strom et al. (1981) and Forte et al. (1981), who concluded from their multicolor photometry of the Virgo ellipticals that the globular clusters at a given galactocentric radius were bluer (and by hypothesis more metal-poor) than the halo light at the same location. When combined with the evidence on their extended spatial distribution (sec. 3 above), this was the second major piece of evidence that the clusters might belong to a somewhat older population than the bulk of the halo stars. The clusters might even be responsible for the first phase of metal enrichment for the entire halo (De Young et al. 1983). Shortly after that, it was pointed out (Harris 1983) that just the same color difference between the clusters and the halo light could also be found in the dwarf ellipticals of the Local Group. Multicolor BVI and Gunn-Thuan CCD photometry currently in progress (Cohen 1986; Harris and Allwright 1987) for the GCS's in several Virgo ellipticals should be able to investigate the reality of this effect further.

As the evidence concerning the differences between galactic spheroids (halos) and their embedded cluster systems has accumulated, support has grown for the single explanation that the clusters are the older of the two, even though they make up only a tiny fraction (less than 18) of the total halo light. A summary is as follows:

- Globular cluster systems tend to be more spatially extended than normal $E$ galaxies (sec. 3). For the two known GCS's in $c D$ galaxies, it is interesting that good agreement with the halo structure is seen, but this feature may well be a result of whatever merger or capture events have produced the extended $C D$ structure, as opposed to something arising from the initial formation process.

- Globular cluster systems in some disk galaxies are noticeably rounder in projection than the galaxy halo, and match the radial structure of the halo reasonably well only along the minor axis (sec. 3). - Globular clusters in a wide range of E galaxies (Virgo gE's, Local Group dE's) are bluer (and by hypothesis of lower metallicity) than the halo stars at the same projected galactocentric radius. - The globular cluster system in M 87 appears to form a kinematically hotter subsystem (larger 
velocity dispersion) than the halo light at the same projected radius (Mould et al. 1986; Huchra and Brodie 1986).

If there is a particular, somehow unique, early epoch which is especially suitable to globular cluster formation, then we might envisage that the ellipticals in general got an earlier start in this epoch than the disk galaxies, and that the E's that found themselves in rich environments benefitted even more (Harris 1986). In this way, the observed mean differences in specific frequency (sec. 2), as well as the more extended space distribution, lower metallicity, and kinematics, could all be a natural outcome of the protogalactic collapse as long as this special epoch is earlier than the bulk of the halo star formation by about a free-fall time. The only specific model which gives some theoretical underpinning to such a picture is the recent discussion of Fall and Rees (1985), which gives a plausible reason for the cluster-sized units condensing out significantly earlier than the majority of the material: the gas in smaller clumps or lower-density zones would be prevented by heating from its surroundings from entering gravitational instability and collapse until later on.

\section{THE CENTRAL SUPERGIANTS AND THE CLUSTER FORMATION EPOCH}

Even if we have arrived at a coherent overall description of the evolutionary role of GCS's in normal galaxies, there is more to the story. The keenest recent interest of all has been attracted to the extraordinary properties of the systems that are found in the supergiant ellipticals located at the centers of rich galaxy groups. There are now four such systems known: M 87 in Virgo, NGC 3311 in Hydra I, NGC 1399 in Fornax (Harris 1986, Hanes and Harris 1986), and NGC 4874 in the Coma Cluster (Thompson and Valdes 1987; Harris 1987). These stand apart in only one respect from the "normal" GCS's described above: they have a specific frequency 3 or more times higher than average. Forming the sheer numbers of clusters that are seen in these dominant central galaxies (M 87, for example, probably holds more than 20,000 clusters) will require special conditions of formation over and above anything that has yet been suggested, in the Fall-Rees scenario or elsewhere. Despite some interesting recent attempts to explain such systems by dynamical simulations involving tidally disrupted neighbors (see Muzzio 1986), there does not seem any clear way to arrive at such a system by taking an existing normal galaxy and then capturing swarms of additional clusters (at all radial distances!) to the exclusion of other types of halo stars. It seems necessary to build them in from the start.

The Coma Cluster galaxies deserve special mention here since, at a mean redshift near $7000 \mathrm{~km} / \mathrm{s}$, they are the closest members of an outstandingly rich and dense (Abell class I) cluster. Detecting globular clusters in the two biggest Coma ellipticals, NGC 4874 and 4889, has long been a challenging goal for photometrists in this field. [Comparing the mean redshifts of Coma and Virgo (see table below) shows that Coma should be $\simeq 5.6$ times (or $3.7 \mathrm{mag}$ ) more distant than Virgo. 
If the brightest $M 87$ clusters appear in large numbers for $B>21$, then in Coma they should appear at $B \approx 24.7$ with their peak near $B=28.4$.]

Some modest success in this direction now seems to have been achieved. Thompson and Valdes (1987) have obtained CCD frames with the ISIS camera at the CFHT on Mauna Kea which show a clear excess of starlike images in a small (1') field just off the center of NGC 4874. Harris (1987) has used the RCA1 CCD camera at the CFHT prime focus to obtain deep images of both NGC 4874 and the other Coma supergiant, NGC 4889. The GCS's around both these galaxies do seem to be resolved; preliminary results for their LF's are displayed here in Fig. 4. (It should be emphasized that the fit shown to the M 87/Virgo LF is not a direct distance measurement for Coma, but simply a consistency test that the Coma systems resolve at the magnitude level expected if they are intrinsically similar to the Virgo globular clusters.) Notably, NGC 4889 does not have as large a GCS population as NGC 4874. Comparing the observed numbers in similar areas around each galaxy indicates that their ratio of specific frequencies is $\mathrm{S}(4874) / \mathrm{S}(4889)-3 \pm 1$. The $S$-values themselves are highly uncertain because only the top magnitude of the cluster LF is seen, and a large extrapolation is needed to predict the total population; nevertheless, $N 4874$ clearly has a specific frequency similar to that of M 87 or higher.

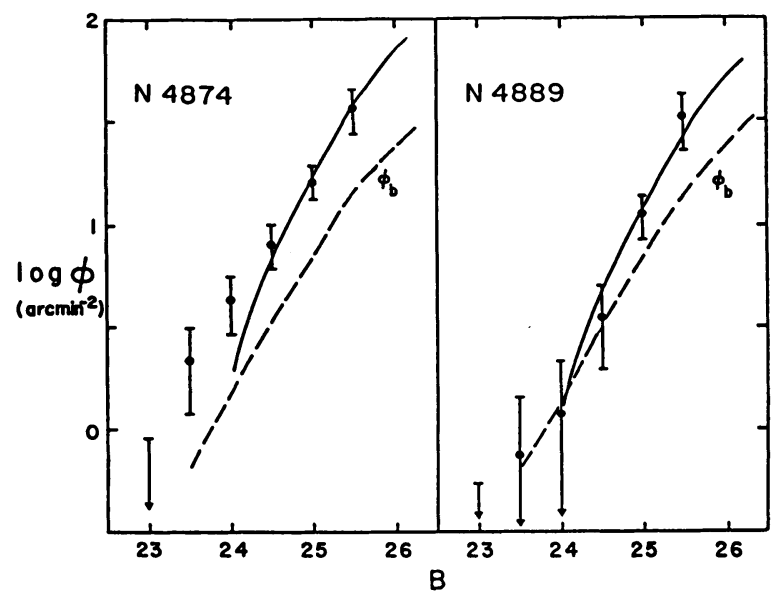

Fig. 4. Preliminary luminosity functions for the globular cluster systems in the supergiant Coma ellipticals, NGC 4874 and 4889 (Harris 1987). Here $\phi$ is the projected number density of measured images near each galaxy (excluding clearly nonstellar images, and corrected statistically for background objects) per quarter-magnitude interval $B$. The solid line represents the M 87/Virgo globular cluster LF, shifted fainter by 3.7 magnitudes and fitted to the data; the dashed line $\phi_{b}$ is the comparison-field LF which was subtracted from the raw data. 
Results for all four central supergiants are summarized in Table II below. Note that in every case, the radial velocity $\left(V_{g}\right)$ of the galaxy is within $1 / 4$ of a standard deviation of the mean velocity $\left(V_{c l}\right)$ of its cluster (in the table, $\Delta \mathrm{V}=\mathrm{V}_{\mathrm{g}}-\mathrm{V}_{\mathrm{cl}}$, expressed in units of the central velocity dispersion of the cluster). In other words, it appears that all these giants are sitting rather quietly enthroned at the dynamical centers of their surroundings. It is important to note that the anomaly of an outstandingly populous GCS appears to be very strongly connected with position: no large galaxies other than the four listed here are known to have it; and conversely, all the giant $E$ galaxies that can clearly be identified as central objects in rich surroundings (and that have been observed so far) do have it.

TABLE II

Globular Cluster Systems in Central Giant Ellipticals*

\begin{tabular}{|c|c|c|c|c|c|}
\hline Object & $\mathrm{M}_{\mathbf{V}}{ }^{\mathrm{T}}$ & $\mathrm{V}_{\mathrm{g}}$ & $\mathrm{v}_{\mathrm{cl}}$ & $|\Delta V|$ & S \\
\hline M $87(N 4486)$ & -22.5 & 1190 & 1000 & $0.23 \sigma$ & 15 \\
\hline NGC 1399 & -20.9 & 1430 & 1460 & $0.06 \sigma$ & 16 \\
\hline NGC 3311 & -22.4 & 3575 & 3420 & $0.17 \sigma$ & 20: \\
\hline NGC 4874 & -22.9 & 7120 & 6950 & $0.14 \sigma$ & 15: \\
\hline
\end{tabular}

* Here $\mathrm{M}_{\mathrm{V}}^{\mathrm{T}}$ is the galaxy luminosity assuming HO $=75 ; \mathrm{S}$ is the specific frequency of the globular cluster system.

Clearly, the story of their formation must run a little differently than for other galaxies. One possibility is perhaps to be found in the comment by Larson (1987) that globular clusters are likely to form within the dense cores of larger, bound units that might have been of dwarf-galaxy size (e.g. $10^{8} \mathrm{M}_{\mathrm{o}}$ or so). Thus the protogalaxies at the centers of big groups would need to have a process which especially encouraged the formation of these dense cores all through the gas-rich proto-halo. It is not unlikely that these same protogalaxies underwent especially early and violent nuclear activity (the quasar phenomenon?), either by being the first objects in their environments to collapse or by suffering a uniquely high interaction rate with the other protogalaxies around it (cf. Stocke and Perrenod 1981; Yee and Green 1984; De Robertis 1985). The resulting shocks moving through the halo gas might then have started proto-globular cores forming in unusual numbers (Gunn 1980; McCrea 1982). At least on a qualitative level, this approach would not conflict with the Fall-Rees picture that heating from the surrounding medium simultaneously prevented the less massive clouds from condensing until their metal abundance was higher or the surroundings were cooler. 
An alternative idea mentioned, e.g., by Fall and Rees, is that heavy gas infall and the development of a cooling flow in these big galaxies allowed their epoch of cluster formation to continue long past the normal galaxy formation stage. This is unlikely, for the reason that the period of cluster formation must have finished clearly before the bulk of the halo started condensing - otherwise, both types of objects would now have the same radial distribution and metallicity, which is contrary to the observations. The existing measurements on radial distribution, specific frequencies, colors and metallicities, and kinematics (see sec. 4 above) for GCS's in large galaxies already place difficult constraints on appropriate models for their formation. It seems necessary to construct a model in which the clusters formed in a rather narrowly defined epoch, clearly before the rest of the halo but after the even more extended "dark" matter, almost independent of galaxy size or type but with some noticeable influence from environment. If we could understand why these remarkable systems exist, we are likely to understand much more about the entire galaxy formation process.

For stimulating ideas related to these topics, I am indebted to $D$. Hanes, R. Larson, R. Pudritz, M. Rees, and numerous other colleagues. M. Hazen and $H$. Liller provided invaluable hospitality during this conference. This work was supported by an NSERC (Canada) operating grant to the author.

\section{REFERENCES}

Blaauw, A. 1965 in Galactic Structure, A.Blaauw and M.Schmidt, eds., U. Chicago Press, Chicago, p.435.

Cohen, J. 1986 preprint.

De Robertis, M. 1985 Astron.J. 90, 998.

De Young, D. S., Lind, K., and Strom. S. E. 1983 Publ. Astron. Soc. Pacific 95, 401.

Fal1, S. M. and Rees, M. J. 1985 Astrophys. J. 298, 18.

Forte, J. C., Strom, S. E. and Strom, K. M. 1981 Astrophys. J. Letters 245, L9.

Geller, M. J., and Huchra, J. P. 1983 Astrophys. J. Supp1.52, 61.

Grillmair, C. J., Pritchet, C. J. and van den Bergh, S. 1986, Astron. J. 91, 1328 .

Gunn, J. E. 1980 in Globular Clusters, D. Hanes and B. Madore, eds., Cambridge Univ. Press, Cambridge, p.301. Hanes, D. A. 1977a Mem. Roy. Astron. Soc. 84, 45; and 180, 309.

Hanes, D. A. $1977 \mathrm{~b}$ Mon. Not. Roy. Astron. Soc. 179, 331.

Hanes, D. A. 1980 in Globular Clusters, D.Hanes and B.Madore, eds., Cambridge Univ. Press, Cambridge, p. 213.

Hanes, D. A. 1987 in IAU Symposium No. 126. Globular Cluster Systems in Galaxies, J. Grindlay and A. G. D. Philip, eds., Reidel, Dordrecht, p. 617.

Hanes, D. A. and Harris, W. E. 1986a Astrophys. J. 304, 599.

Hanes, D. A. and Harris, W. E. 1986b Astrophys. J. 309 (in press).

Hanes, D. A. and Harris, W. E. 1987 in preparation. 
Harris, G. L. H., Hesser, J. E., Harris, H. C. and Curry, P.J. 1984 Astrophys. J. 287, 175.

Harris, H. G., Bothun, G. D. and Hesser, J. E. 1987 in IAU

Symposium No, 126, Globular Cluster Systems in Galaxies, J. E.

Grindlay and A. G. D. Philip, eds., Reidel, Dordrecht, p. 613.

Harris, W. E. 1981 Astrophys. J, 251, 497.

Harris, W. E. 1983 Publ, Astron. Soc. Pacific 95, 21.

Harris, W. E. 1986 Astron. J. 91, 822.

Harris, W. E. 1987, preprint.

Harris, W. E. and Allwright, J. W. B. 1987 in preparation.

Harris, W. E., Harris, H. C. and Harris, G. L. H. 1984 Astron. J. $89,216$.

Harris, W. E. and Racine, R. 1979 Ann. Rev, Astron, Astrophys, 17, 241.

Harris, W. E. and Smith, M. G. 1976 Astrophys. J. 207, 1036.

Harris, W. E., Smith, M. G. and Myra, E. S. 1983 Astrophys. J. 272, 456.

Harris, W. E. and van den Bergh, S. 1981 Astron. J. 86, 1627.

Hubble, E. 1932 Astrophys. J. 76, 44.

Huchra, J. P. and Brodie, J. 1986 preprint.

Huchra, J. P. and Geller, M. J. 1982 Astrophys. J. 257, 423.

Larson, R. 1987 in IAU Symposium No. 126, Globular Cluster Systems in Galaxies, J. Grindlay and A. G. D. Philip, eds., Reidel, Dordrecht, p. 311 .

Lauer, T. R., and Kormendy, J. 1986 Astrophys. J. Letters 303, L1.

McCrea, W. H. 1982 in Progress in Cosmology, A. Wolfendale, ed., Reidel, Dordrecht, p.239.

Mould, J. R., Oke, J. B. and Nemec, J. M. 1986 Astron. J, (in press).

Muzzio, J. C. 1986 Astrophys. J, 301, 23.

Muzzio, J. C., Martinez, R. E. and Rabolli, M. 1984 Astrophys. J. 285,7 .

Oemler, A. 1976 Astrophys. J. 209, 693.

Racine, R. 1980 in Star Clusters, IAU Symposium No. 85, J.E.Hesser, ed., Reide1, Dordrecht, p.369.

Shapley, H. 1918 Astrophys. J. 48, 154.

Stocke, J. T. and Perrenod, S. C. 1981 Astrophys. J. 245, 375.

Strom, S. E., Forte, J. C., Harris, W. E., Strom. K. M., Wells, D. C. and Smith, M. G. 1981 Astrophys, J. 245, 416.

Strom, S. E. and Strom, K. M. 1978 Astron. J. 83, 732 .

Thompson, L. A. and Valdes, F. 1987 preprint.

Tremaine, S. 1976 Astrophys. J. 203, 345.

van den Bergh, S. 1977 Vistas Astron, 21, 71.

van den Bergh, S. 1985 Astrophys. J. 297, 361.

van den Bergh, S., Pritchet, C. J. and Grillmair, C. J. 1985, Astron. J. 90, 595.

Yee, H. K. C. and Green, R. F. 1984 Astrophys. J. 280, 79. 


\section{DISCUSSION}

ZINN: It appears to me that most, if not all, of the evidence that the globular clusters constitute a more distended and bluer population than the halo stars comes from observations of elliptical galaxies. Most of the evidence that globular clusters and halo stars are very similar in metallicity, spatial distribution, and kinematics comes from observations of one disk galaxy, the Milky Way. Is there an example of a disk galaxy in which the globular cluster population is definitely more distended and bluer than the population of halo stars?

HARRIS, W.: As far as the metallicity of the halo population is concerned, there are no other disk galaxies aside from the M 31 and the Milky Way for which metallicities or photometric colors are available yet. For the space distributions, H. Harris and J. Hesser show that the clusters around NGC 7814, an edge-on Sa disk galaxy, form a more extended space distribution than the halo light; and as far as NGC 4594 and 3115 are concerned, the cluster distribution agrees with the light distribution reasonably well only along the minor axis, which really suggests that the cluster system is intrinsically more extended.

SCHOMMER: I am rather worried about a similar problem. The brightest outer cluster in $M 33$ has $(B-V)=0.51\left((B-V)_{0}<0.45\right)$. I think this is a massive intermediate age cluster and not a true globular cluster, color measures are clearly important; the differences Strom et al. noted could easily be age and not metallicity. There is some, admittedly controversial, evidence that $\mathrm{E}$ galaxies have an intermediate age stellar population.

HARRIS, W.: You've noticed that in my summary I neglected the Sc and irregular galaxy systems; in the later-type galaxies with bigger halos that problem is probably less severe. However, it does point up the need for more color information in all these systems.

OSTRIKER: Many of the differences that you find between the elliptical light distribution and the globular cluster system may be due to evolution of the cluster system. In centrally concentrated galaxies the inner clusters are very effectively destroyed.

HARRIS, W.: Most of the statement that the radial distribution of the clusters vs. the halo stars are different relies on the observed regions more than $10 \mathrm{kpc}$ from the galaxy center and not the nuclear region. It will be interesting to see if the destruction mechanisms are effective that far out, or farther.

KING: This question is meant with a spirit of caution rather than challenge. How can you be sure of a Gaussian fit when there is only one point on the other side of the peak? 
HARRIS, W.: My comments about the "Gaussian" nature of the distribution were pragmatic ones rather than any statement about the true shape of the whole distribution. For example, vandenBergh (1985 Astrophys. J.) has suggested that the luminosity function for the galactic globular clusters is somewhat asymmetric when the faint half is included. However, the clusters fainter than the peak point are somewhat irrelevant to the distance fitting problem since they are never seen in the more distant galaxies. That means that we're primarily interested in the shape of the brighter half alone, which is why I showed the fit to the Virgo luminosity function the way I did.

BAUM: You called attention to the unusually high specific frequencies of globular clusters associated with central galaxies in four clusters (including NGC 4874 in Coma) and you mentioned the "stationarity" of those galaxies (judged from radial velocities) relative to the rest of the cluster. Yet in the Coma Cluster there are two central galaxies, so neither may be a unique stationary infall locus. The other central galaxy in Coma is NGC 4889. Do you have globular cluster data for NGC 4889? Is it different from NGC 4874?

HARRIS, W.: Yes, I have new CCD data (from the CFHT this March) for both NGC 4874 and 4889 . The key result is that 4874 seems to have about $3 \pm 1$ times as many clusters (excess images) than 4889, which would make it analogous to the $M 87 / M 49$ situation in Virgo. The velocity of NGC 4889 is a few hundred $\mathrm{km} / \mathrm{sec}$ farther away from the Coma mean than NGC 4874, and also NGC 4874 is the center of a bright radio source, so on balance it looks as if NGC 4874 is more likely to be the central object.

CAYREL: You have used a specific number per unit of visual absolute magnitude. Is it true that $S$ would be more stable versus the morphological type if defined with respect to something more related to the mass of the galaxy?

HARRIS, W.: A specific frequency defined relative to mass instead of total light might well be more interesting, but would be harder to define, since the mass distribution is so much more extended than the galaxy light and it's not clear how you would cut it off at some radius to get a total mass that was meaningful. Getting a total luminosity for just the halo (e.g in a disk galaxy) is difficult too, but we haven't been able to think of anything better to use as yet.

HANES: I refer Ivan King to my poster paper, where I show that one can fit Gaussians to truncated data sets with remarkable confidence via maximum likelihood methods. It is time that some people interested in $\mathrm{H}$ o have alternatively supported and then criticized the use of globular clusters as distance indicators, but let me record here that de Vaucouleurs has always been very positive about their use ever since he first used them in his 1977 Nature paper. 
ROBERTS: You've described globular clusters as having a Gaussian like luminosity function. It is worth noting that the luminosity functions for stars in our galaxy, galaxies themselves, and clusters of galaxies do not have such a peaked shape. If there is a universal luminosity function we can surely learn much from these differences.

HARRIS, W.: As you suggest, the luminosity function of the clusters as we see them now is a combination of how they formed plus what has happened since then to destroy them selectively. So we should be able to learn about both processes. If we really want to use globular clusters as distance indicators, we need to do a lot more work in understanding the detailed similarities and/or differences from one galaxy to another. The main difference here compared with the other indicators like Cepheids and supergiants is that the people working on those other indicators have spent the time to find out what the first and second order characteristics of their standard candles actually are like from one galaxy to another. Because of the lack of data for enough galaxies to enough depth, we have not got that far with the globular clusters so far. 